\title{
DELAUNAY-SUPPORTED EDGES FOR IMAGE GRAPHS
}

\author{
Nicholas Dahm ${ }^{\star} \quad$ Yongsheng Gao ${ }^{\star} \quad$ Terry Caelli ${ }^{\dagger} \quad$ Horst Bunke \\ ${ }^{\star}$ School of Engineering, Griffith University, Brisbane, Australia \\ $\{$ n.dahm, yongsheng.gao $\} @$ griffith.edu.au \\ ${ }^{\dagger}$ Electrical and Electronic Engineering, University of Melbourne, Australia \\ tcaelli@unimelb.edu.au \\ ${ }^{\ddagger}$ Institute of Computer Science and Applied Mathematics, University of Bern, Switzerland \\ bunke@iam.unibe.ch
}

\begin{abstract}
Graphs are a powerful and versatile data structure for pattern recognition. However, their flexibility brings inherent complexities for algorithms which seek to create or utilise graphs. In the context of computer vision, many feature detection algorithms can extract a suitable vertex set from an image. The creation of edges between these vertices presents new challenges, and effective methods for edge creation only exist for certain types of vertices such as points and regions. This paper presents a novel method for creating edges for image graphs, while supporting a wide array of vertex types. The presented method is principled, and its robustness is shown experimentally against a number of affine and projective transforms, as well as noise.
\end{abstract}

Index Terms - graph matching, computer vision

\section{INTRODUCTION}

The characterisation of image features and their interrelationships is an important topic in structural pattern recognition. For such a task, the expressive power of graphs holds a distinct advantage over simpler data structures such as vectors, strings, and trees. However, the flexibility of graphs provides extra challenges when creating principled methods for their creation and utilisation. These challenges, coupled with the relatively high computational cost of graph matching algorithms, have prevented graphs from becoming as widely used as vectors.

One key obstacle in using graphs in computer vision is conversion of an image into a graph. Creating a vertex set from an image is straightforward, using existing feature detectors. Common features used are keypoints, line segments, polylines, polygons, and regions. The selected feature type determines which graph edge generation techniques can be effectively applied.

In the case of regions, the most common technique is to create edges between adjacent regions, creating a region ad- jacency graph[1]. These edges have a clear definition as to when they will be created, and what they characterise. An added benefit is that these graphs are connected (not disjoint) planar graphs.

For points, Delaunay triangulation (DT) is the most common edge generation technique. The resulting graph is a connected planar triangulated mesh, where edges signify spatial closeness. Delaunay triangulation seeks to create a triangulated mesh where no points lie within the circumcircle of each triangle. This discourages long and thin triangles, making it well-suited for 3D modelling[2], as well as being aesthetically pleasing.

The remaining feature types do not have well-established methods for graph edge generation. As such, when transformed into graphs, they are often made to be fully connected (complete) graphs[3,4]. The edge properties in these complete graphs can describe complex relational properties between vertices. However, being a complete graph, the graph topology itself does not provide any information.

If a non-complete graph is preferable, DT can be used by taking a representative point (often midpoint) of features. Such an approach can yield good results when the space between figures is large, relative to the size of the features. However, when features are large, cluttered, or encapsulating each other, a single point cannot effectively represent them.

In this paper we propose a novel method which can create edges for image graphs in a robust and principled manner. Our method supports using any of the above features as vertices, including different feature types for different vertices.

The remainder of the paper is organised as follows. Section 2 introduces our method, which creates Delaunaysupported graphs. In Section 3, performance characteristics of our method are shown, with comparisons against the traditional DT method. Finally, Section 4 examines the strengths of the algorithm, and draws a number of conclusions from the results. 


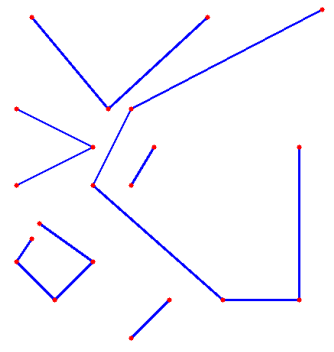

(a) Graph Vertices

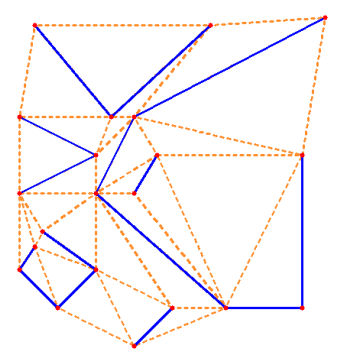

(b) Constrained Triangulation
Fig. 1: Triangulation process, including points (red), segments (blue), and links (orange/dashed)

\section{DELAUNAY-SUPPORTED GRAPH}

A Delaunay-supported graph (DSG) is a two-layer image graph (not to be confused with a dual-graph[5]), consisting of a (top) graph layer, and a (lower) triangulation layer. The interaction between these two layers allows the graph edges to be robust against transformations and noise.

Before a DSG can be created, a set of graph vertices must be provided. Each graph vertex is comprised of one or more points, and zero or more line segments between those points.

Definition 1. A (Delaunay-supported graph) vertex is a pair $v_{i}=\left(P_{i}, S_{i}\right)$, where $P_{i}=\left\{p_{1}, \ldots, p_{k}\right\}$ is a non-empty set of points, and $S_{i} \subseteq P_{i} \times P_{i}$ is a (possibly empty) set of segments.

This allows each vertex to represent any of the aforementioned features, plus more complex features. Technically, the configuration of points and segments in a single vertex can represent any simple (possibly disconnected) graph, embedded in a 2D plane (although not necessarily planar). However, to aid in illustrations, we restrict vertices to polylines in this paper, as can be seen Fig. 1a.

The points and segments from graph vertices are used as inputs to generate the triangulation layer, as shown in Fig. 1b. This triangulation layer is not a true Delaunay triangulation, but instead a constrained Delaunay triangulation (CDT)[6]. In a traditional DT, a number of points are provided, and the DT algorithm creates a number of links between those points, forming a triangulated mesh. A CDT is similar to a DT, but also includes a number of segments (enforced links), which must be included in the final triangulation.

Definition 2. Let $V$ be the vertices of a Delaunay-supported graph. A constrained Delaunay triangulation is a triple $T=$ $(P, S, L)$, where $P=\cup\left\{P_{i}:\left(P_{i}, S_{i}\right) \in V\right\}$ is a set of points, $S=\cup\left\{S_{i}:\left(P_{i}, S_{i}\right) \in V\right\}$ is a set of segments (enforced links), and $L=\left\{l_{1}, \ldots, l_{k}\right\}$ is a set of (unenforced) links which are created by the CDT algorithm.

If two points are connected by a link in the triangulation
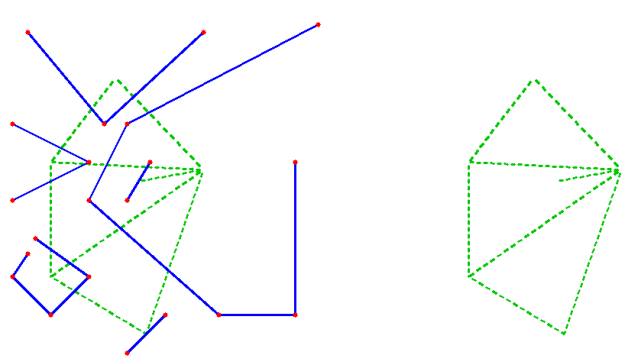

(a) Delaunay-supported graph

(b) Delaunay-supported edges

Fig. 2: The graph edges (green/dashed), created from the triangulation in Fig. 1b, shown with and without vertices

layer, a Delaunay-supported edge is created in the graph layer between the vertices which those points belong to.

Definition 3. A Delaunay-supported edge is an unordered pair $e=\left\{v_{i}, v_{j}\right\}$, which is supported by at least one link $l=\left\{p_{m}, p_{n}\right\}$, where $p_{m} \in P_{i}$ and $p_{n} \in P_{j}$.

Duplicate edges and self-loops which would be created by this rule can either be ignored (as we will do in this paper) or utilised to create a multigraph. An illustration of the final graph is given in Fig. 2a, the edges of which were created using the regular links from Fig. $1 b$.

Definition 4. A Delaunay-supported graph is a triple $D S G=$ $(V, E, T)$, where $V=\left\{v_{1}, \ldots, v_{n}\right\}$ is a set of vertices, $E \subseteq V \times V$ is a set of Delaunay-supported edges, and $T$ is a constrained Delaunay triangulation.

For comparison, an example graph created using the traditional DT edge method is shown in Fig. 3. In both Figs. 2 and 3, the edges are drawn as if connecting the midpoints of graph vertices. For traditional DT edges (Fig. 3) this is accurate, however for Delaunay-supported edges (Fig. 2) this is simply for illustration purposes, as the DSG algorithm will
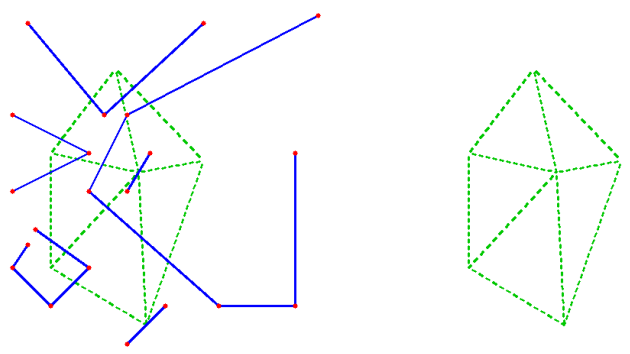
(a) Traditional DT graph
(b) Traditional DT Edges

Fig. 3: The graph edges created when using the traditional DT edge creation method 
not reduce any vertex to a single point (of course, any vertex which began as a single point will stay as such).

Comparing these figures, it is evident that some vertices, whose polylines almost touch, are connected in the DSG, but not in the traditional DT graph. In this example, this issue is due to the midpoint of the longest polyline not being an accurate representation of the whole vertex.

\section{EXPERIMENTATION}

Image graphs, such as those created by the DSG algorithm, are useful for many computer vision tasks, such as image classification and matching. Such tasks often have to deal with changes in pose and illumination. Pose changes warp the spatial geometry of the images, while illumination changes can introduce noise in the feature detection stage.

In these experiments we simulate these conditions, and observe the effects on the graph topology. Pose changes are simulated by performing affine or projective transforms on the graph vertices, while noise is simulated by removing points from the vertices. By performing the transform operations on the vertices instead of the underlying image, any bias caused by errors in the feature detection stage is removed.

\subsection{Implementation}

To evaluate the robustness of DSG, we have implemented both the DSG algorithm, as well as a traditional DT algorithm. The traditional algorithm will take a representative point (midpoint) for each vertex and create graph edges based on the DT of those points.

The DSG algorithm has been implemented in $\mathrm{C}++$, and is provided free to the community ${ }^{1}$. The core graph mechanics are implemented using the Boost Graph Library, while constrained Delaunay triangulation is provided by the Triangle library[2].

To provide features in our experiments, we use the line edge map (LEM) algorithm[7], which extracts polylines from detected image edges.

\subsection{Performance Criterion}

Given that both algorithms differ only in their edge generation, we evaluate their performance in terms of edge changes. The edge retention of a graph is defined as the number of edges which it retains after being transformed, as a fraction of the total number of edges it has.

Definition 5. Let $G_{1}$ and $G_{2}$ be two graphs, created from an original vertex set $V_{1}$ and its transformed counterpart $V_{2}$, respectively. The edge retention percentage between $G_{1}$ and $G_{2}$ is defined as:

$$
\frac{2 \times\left|E_{1} \cap E_{2}\right|}{\left|E_{1}\right|+\left|E_{2}\right|}
$$

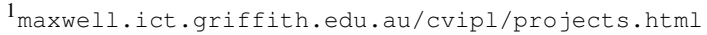

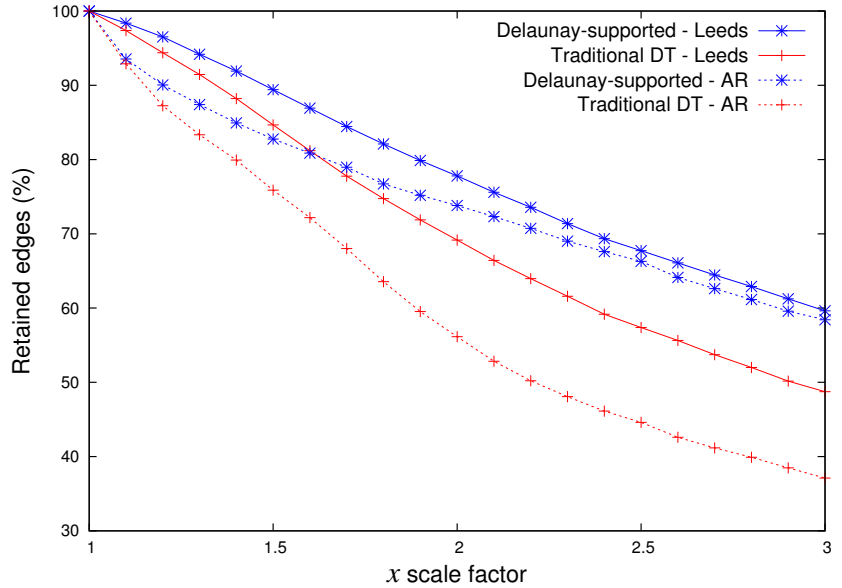

Fig. 4: Edge retention under non-uniform scaling

\subsection{Datasets}

The images used for these experiments will be from the Leeds butterfly dataset[8] and AR Face Database[9], both of which are publicly available ${ }^{2,3}$. From the Leeds dataset, the first 10 images of each butterfly class are taken, giving a total of 100 images. From the AR database, the first 10 images are taken from each expression type in the first session, giving 130 images.

\subsection{Results}

As both edge-generation methods are invariant to (in-plane) rotation, translation, and (uniform) scaling, we test them against the more complex parts of affine and projective transforms.

The first set of results, in Fig. 4, shows the edge retention of both algorithms, under non-uniform scaling $(x=x *$ scale). This spreads out the points horizontally, forcing many horizontal links in the triangulation to flip to being vertical, as the vertical distance is now (relatively) smaller. In this test, DSG retained $6 \%$ more edges at an $x$ scale of $1.5 \times$, increasing to $10-22 \%$ at $2.5 \times$, which is maintained thereafter.

Next, in Fig. 5, we transform the points using a shear $(x=x+(y *$ shear $))$. This transform has a greater effect on triangulations, as many vertical links change which points they are attached to. Once again the DSG algorithm maintains a significant advantage over traditional DT, near-linearly increasing from $7 \%$ at 0.5 to $20 \%$ at 2 . One significant advantage of DSG here is that even though vertical links regularly switch between points, a DSG edge $\left\{v_{i}, v_{j}\right\}$ only requires a single point from $v_{i}$ be close to a single point from $v_{j}$.

For the next test, we move on to projective transforms, rotating points about the $x$-axis, with results shown in Fig. 6.

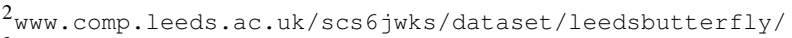

3 www2.ece.ohio-state.edu/ aleix/ARdatabase.html
} 


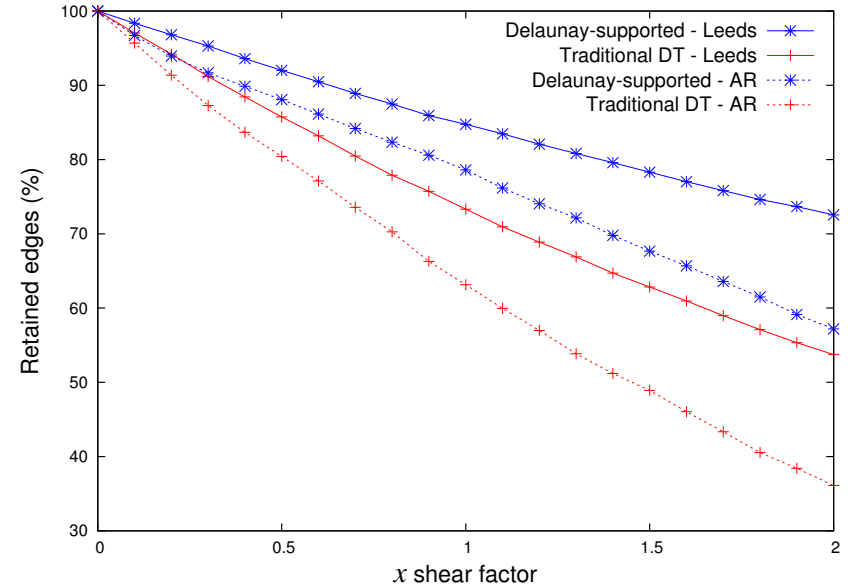

Fig. 5: Edge retention under $x$-axis shearing

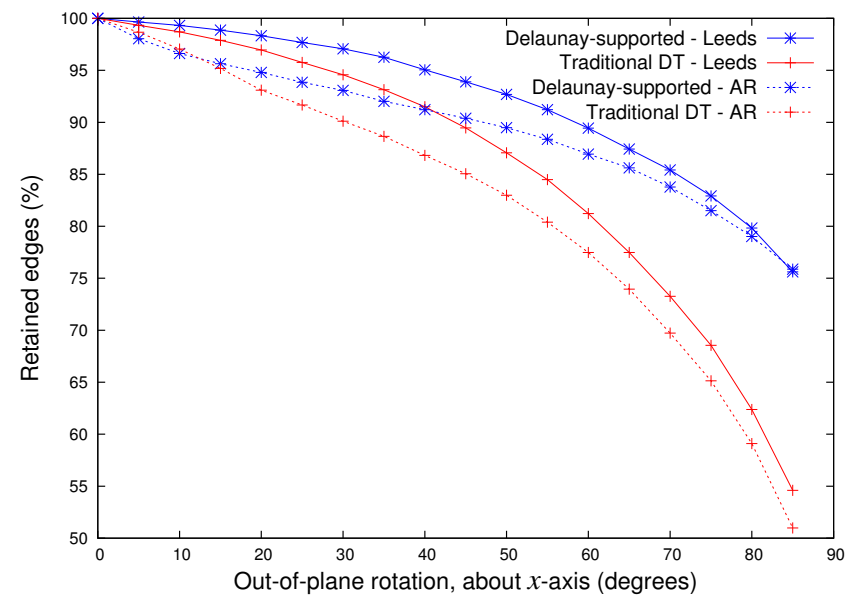

Fig. 6: Edge retention under perspective transform

Such out-of-plane rotation affects point positions exponentially, and leads to a degenerate triangulation at $90^{\circ}$ (every point lies on the $x$-axis). As expected, the edge retention of both algorithms decreases exponentially, with DSG maintaining an exponential lead over traditional DT, with 5\% more edges retained at $45^{\circ}$, increasing to over $20 \%$ at $85^{\circ}$

Lastly, we examine the edge retention of both methods when noise is present in the feature-detection stage. We simulate this noise by deleting random points from the graph vertices. To ensure that no vertices are completely removed, when a vertex has only one point remaining, it is excluded from the pool of deletion candidates. This leads to a case where, after $100 \%$ of the points are deleted (excluding one point from each vertex), we are left with the Delaunay triangulation of the same set of points, regardless of whether DSG or the traditional DT algorithm is used. As Fig. 7 shows, both methods are very resistant to such noise, retaining around $80 \%$ of the edges when $50 \%$ of the points removed.

With up to $80 \%$ of points removed, DSG retains a small

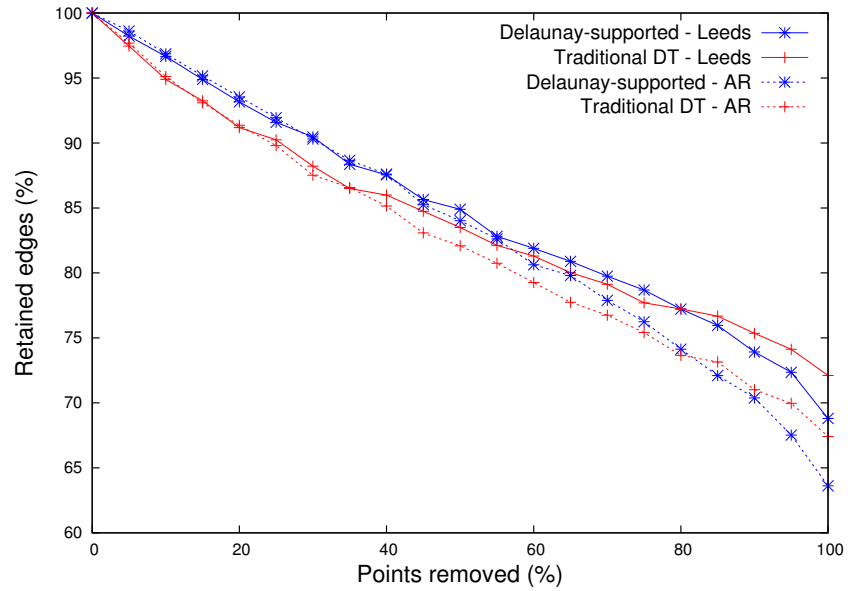

Fig. 7: Edge retention under point removal

but noticeable advantage over traditional DT. This is because the vertex midpoint shift in traditional DT during point deletion is more significant than the relocation of that point's triangulation links is for DSG.

However, after more than $80 \%$ of points are removed, this lead is conceded to traditional DT. As the percentage of points deleted approaches $100 \%$, the topology of DSG is transformed towards that of traditional DT. This change in topology counteracts the edge robustness of DSG, and overpowers it at this $80 \%$ mark.

\section{CONCLUSION}

In this paper we have presented a novel approach to creating edges for image graphs. Our method is applicable to image graphs where vertices are represented by points, line segments, polylines, polygons, or any other configuration of one or more points, with zero or more lines. The ability of our method to effectively handle different vertex types within one graph gives additional flexibility to applications.

We have shown the robustness of the edges created, under both affine and projective transforms. In these results, our method retained significantly more edges than the traditional DT method (up to $25 \%$ more in the projective test). Additionally, in the noise test, our method outperformed the traditional DT method until $80 \%$ of points were removed, despite the significant topological change involved.

It should be noted that, given a set of non-point vertices, the graph created by our algorithm may not be planar, which may affect the computational complexity of certain matching algorithms.

Lastly, we have provided the full $\mathrm{C}++$ source code for our algorithm, to aid the utilisation, and improvement upon, our algorithm. 


\section{REFERENCES}

[1] T. Pavlidis, Structural Pattern Recognition, SpringerVerlag, New York, 1977.

[2] Jonathan Richard Shewchuk, "Triangle: Engineering a 2d quality mesh generator and delaunay triangulator," in $\mathrm{Ap}$ plied Computational Geometry Towards Geometric Engineering, Ming C. Lin and Dinesh Manocha, Eds., vol. 1148 of Lecture Notes in Computer Science, pp. 203-222. Springer Berlin Heidelberg, 1996.

[3] Nicholas Dahm, Yongsheng Gao, Terry Caelli, and Horst Bunke, "Matching non-aligned objects using a relational string-graph," in Proceedings of the IEEE International Conference on Image Processing, Melbourne, Australia, 2013, pp. 3394-3398.

[4] T. Bailey, E.M. Nebot, J.K. Rosenblatt, and H.F. DurrantWhyte, "Data association for mobile robot navigation: a graph theoretic approach," in Proceedings of the IEEE In- ternational Conference on Robotics and Automation, San Francisco, CA, USA, 2000, vol. 3, pp. 2512-2517.

[5] Walter G. Kropatsch, "Building irregular pyramids by dual graph contraction," Tech. Rep., Technical University of Vienna, 1994.

[6] L. Paul Chew, "Constrained delaunay triangulations," Algorithmica, vol. 4, no. 1-4, pp. 97-108, 1989.

[7] Yongsheng Gao and Maylor K. H. Leung, "Face recognition using line edge map," IEEE Transactions on Pattern Analysis and Machine Intelligence, vol. 24, no. 6, pp. 764-779, June 2002.

[8] Josiah Wang, Katja Markert, and Mark Everingham, "Learning models for object recognition from natural language descriptions," in Proceedings of the British Machine Vision Conference, 2009.

[9] A. M. Martinez and R. Benavente, "The AR face database," CVC Technical Report \#24, June 1998. 